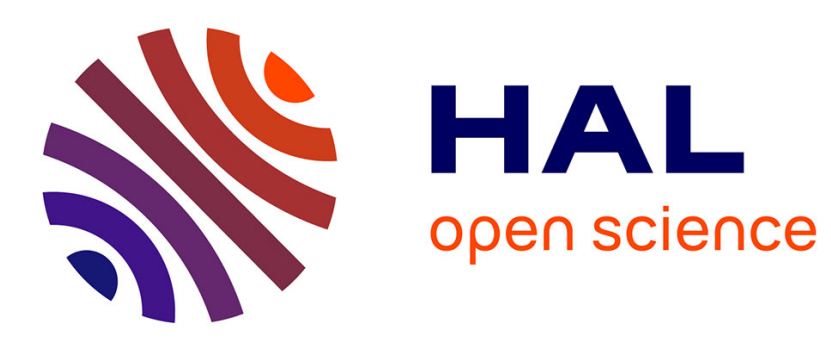

\title{
Bulky Barbiturates as Non-toxic Ionic Dye Insulators for Enhanced Emission in Polymeric Nanoparticles
}

Bohdan Andreiuk, Ilya O Aparin, Andreas Reisch, Andrey S Klymchenko

\section{To cite this version:}

Bohdan Andreiuk, Ilya O Aparin, Andreas Reisch, Andrey S Klymchenko. Bulky Barbiturates as Non-toxic Ionic Dye Insulators for Enhanced Emission in Polymeric Nanoparticles. Chemistry - A European Journal, 2021, 10.1002/chem.202101986 . hal-03284795

\section{HAL Id: hal-03284795 \\ https://hal.science/hal-03284795}

Submitted on 12 Jul 2021

HAL is a multi-disciplinary open access archive for the deposit and dissemination of scientific research documents, whether they are published or not. The documents may come from teaching and research institutions in France or abroad, or from public or private research centers.
L'archive ouverte pluridisciplinaire HAL, est destinée au dépôt et à la diffusion de documents scientifiques de niveau recherche, publiés ou non, émanant des établissements d'enseignement et de recherche français ou étrangers, des laboratoires publics ou privés. 
Bulky Barbiturates as Non-toxic Ionic Dye Insulators for Enhanced Emission in Polymeric Nanoparticles

\author{
Dr. Bohdan Andreiuk, Dr. Ilya O. Aparin, Dr. Andreas Reisch, Dr. Andrey S. \\ Klymchenko* \\ Laboratoire de Bioimagerie et Pathologies, UMR 7021 CNRS, ITI Chimie des Systèmes \\ Complexes, Université de Strasbourg, 74 route du Rhin, 67401, Illkirch, France \\ *Corresponding author: andrey.klymchenko@unistra.fr \\ Twitter: @aklymchenko
}

\begin{abstract}
Bulky hydrophobic counterions (weakly coordinating anions) can insulate ionic dyes against aggregation-caused quenching (ACQ) and enable preparation of highly fluorescent dye-loaded nanoparticles (NPs) for bioimaging, biosensing and light harvesting. Here, we introduce a family of hydrophobic anions based on fluorinated $C$-acyl barbiturates with delocalized negative charge and bulky non-polar groups. Similarly to fluorinated tetraphenylborates, these barbiturates prevent ACQ of cationic dye alkyl rhodamine B inside polymer NPs made of biodegradable poly(lacticco-glycolic acid) (PLGA). Their efficiency to prevent ACQ increases for analogues with higher acidity and bulkiness. Their structure controls dye-dye communication, yielding bright NPs with on/off switching or stable emission. They enhance dye encapsulation inside NPs, allowing intracellular imaging without dye leakage. Compared to fluorinated tetraphenylborates known as cytotoxic transmembrane ion transporters, the barbiturates display a significantly lower cytotoxicity. These chemically available and versatile barbiturate derivatives are promising counterion scaffolds for preparation of bright non-toxic fluorescent nanomaterials.
\end{abstract}

Keywords: nanoparticles, fluorescence, dyes/pigments, bulky hydrophobic anions, aggregation. 


\section{Introduction}

Fluorescent nanoparticles (NPs) gained importance in recent years due to their unique optical properties and numerous potential applications, including biological imaging and biosensing ${ }^{[1]}$ as well as light harvesting. ${ }^{[1 \mathrm{~b}, 2]}$ Organic fluorescent nanoparticles are of particular interest as the highly versatile nature of organic materials and fluorescent dyes enable preparation of materials of programmed optical properties, easy functionalization with targeting ligands, antibodies, DNA, stealth groups, etc. On the other hand, bio-applications of organic nanoparticles impose strict requirements such as biocompatibility, biodegradability, low toxicity and eco-friendliness, which remains a challenge even for organic nanomaterials. Important examples of organic NPs include AIE NPs,${ }^{[3]}$ conjugated polymer NPs, ${ }^{[4]}$ dye-loaded polymeric NPs ${ }^{[3 b, 5]}$ and lipid NPs. ${ }^{[6]}$ Dyeloaded polymeric NPs are particles of choice in view of bio-applications, because they present several unique features. On the one hand, by selecting appropriate polymer matrix, NPs with desired biocompatibility, biodegradability and stability can be obtained. For example, poly(lacticco-glycolic acid) (PLGA), approved for human use by the United States Food and Drug Administration and by the European Medicine Agency, can be used to prepare biodegradable NPs, which are generally known in the field of drug delivery. ${ }^{[7]}$ On the other hand, selection of the dye and its loading level allow fine tuning of optical properties of NPs, such as absorption and emission color, ${ }^{[8]}$ brightness, ${ }^{[9]}$ photostability, ${ }^{[10]}$ etc. ${ }^{[5]}$

However, preparation of bright dye-loaded NPs requires addressing two key challenges. First, fluorescent dyes should be efficiently encapsulated into the polymer matrix without dye leakage (leaching) in biological media. ${ }^{[1]}$ Second, at high dye loading aggregation-caused quenching (ACQ) is commonly observed, which complicates preparation of bright NPs. ${ }^{[5 a]}$ Dye self-quenching is generally caused by the flat aromatic structure of fluorophores, which favors pistacking of dyes into so-called H-aggregates with face-to-face assembly. ${ }^{[12]}$ To address the problem of ACQ, a number of approaches have been proposed, ${ }^{[5 a]}$ which include aggregationinduced emission, ${ }^{[3 a, 13]}$ the use of bulky side groups, ${ }^{[10,14]}$ and bulky hydrophobic counterions. ${ }^{[15]}$ Our previous works showed that bulky hydrophobic counterions are of particular interest for dyeloaded polymeric NPs, as they can address both the problems of ACQ and dye leakage from NPs for different cationic dyes. ${ }^{[8,11 \mathrm{~b}, 15 \mathrm{a}]}$ These counterions are generally weakly coordinating anions, most commonly tetraphenylborate (TPB) derivatives. ${ }^{[16]}$ They are characterized by large size, high hydrophobicity and strong delocalization of the negative charge over the large volume of the anion. Weakly coordinating anions are often constituents of ionic liquids and they are commonly used for stabilization of reactive cations ${ }^{[17]}$ and for preparation of ion-selective electrodes and electric batteries. ${ }^{[16,18]}$ Earlier works showed that they can minimize self-quenching in pure dye salts, ${ }^{[19]}$ and the most efficient were those characterized by large size and high level of fluorination. ${ }^{[20]}$ As 
we showed later on, these counterions can form highly hydrophobic ion pairs with a cationic dye, which ensures its nearly quantitative encapsulation inside the hydrophobic polymeric matrix even at high loading during nanoparticle synthesis by nanoprecipitation. ${ }^{\left[11 \mathrm{~b},{ }^{21]}\right.}$ Moreover, these bulky anions serve as spacers (or insulators) between encapsulated cationic dyes, thus preventing their self-quenching through pi-stacking into H-aggregates. ${ }^{[11 b, 15 a, 22]}$ Therefore, despite very high loading of cationic dyes with these bulky hydrophobic anions (up to $50 \mathrm{wt} \% \mathrm{vs}$ polymer), the obtained NPs exhibited unprecedentedly high fluorescence quantum yields $(\sim 50 \%) .{ }^{[23]}$ In this way it was possible to obtain NPs 6-100 fold brighter than quantum dots of similar size and emission color. ${ }^{[9,15 a, 24]}$ Owing to the short dye-dye distances inside these highly loaded NPs, the loaded dyes exhibited unique collective behavior due to coupling by ultrafast excitation energy transfer, ${ }^{[15 a]}$ which enabled preparation of a giant light-harvesting nanoantenna for single-molecule detection at ambient light. ${ }^{[25]}$ Moreover, the concept of bulky counterions works with both rhodamine and cyanine dyes, which enabled preparation of differently colored ultrabright NPs and development of a cell barcoding system for in vitro and in vivo applications. ${ }^{[8]}$ Such NPs have also been used for the assembly of biosensors for detection of nucleic acids with single-molecule sensitivity ${ }^{[23-24]}$ and live tracking inside mouse brain. ${ }^{[26]}$ However, so far, the counterion approach was mainly limited to fluorinated tetraphenylborate derivatives ${ }^{[8,11 b, 15 a]}$ and an aluminate of perfluorinated tert-butanol. ${ }^{[21]}$ These ions can transport cations, including protons, through biological membranes, such as cell plasma membranes and mitochondria, which leads to cytotoxicity at micromolar concentrations. ${ }^{[27]}$ Moreover, bulkier and more fluorinated anions were found to be more cytotoxic because of their stronger capacity to transport cations. ${ }^{[27]}$ Even though dye-loaded polymeric NPs encapsulating these counterions were not cytotoxic, ${ }^{[8,21]}$ questions can be raised about potential toxicity of these anions after biodegradation of the polymer matrix. Moreover, due to the symmetric nature of these anions, it is synthetically challenging to monofunctionalize them. ${ }^{[28]}$ Therefore, there is a need to look for alternative scaffolds for a hydrophobic ion. One approach, called small-molecule ionic isolation lattices (SMILES) proposed to generate bulky hydrophobic anions by complexing small inorganic anions with a large macrocycle (cyanostar), which allows to significantly decrease ACQ of cationic dyes in bulk polymeric materials ${ }^{[15 b]}$ and nanoparticles. ${ }^{[29]}$ Another attractive approach is the design of an organic ion with high charge delocalization ("soft" ion), which would be also highly hydrophobic, similarly to weakly coordinating anions based on borates and aluminates, but without their limitations. Classical examples of hydrophobic anions are oxonol dyes (Figure 1A), in which a single negative charge is delocalized over a long conjugation system. ${ }^{[30]}$ However, oxonol dyes are colored, which would alter or quench the emission of a cationic dye in the counterion-based approach. Therefore, an oxonol analogue with a much shorter conjugation should be developed. 
In the present work, we synthesized a family of organic anions based on a barbiturate scaffold and studied their capacity to prevent ACQ in dye-loaded polymeric NPs. Their weakly acidic methylene group was $C$-acylated in order to enable formation of the anion with delocalized negative charge after deprotonation (Figure 1, A,B), similarly to oxonols, but without absorption of light in the visible region. Our results showed that acylated barbiturates with perfluorobenzoyl group can form stable ion pairs with a cationic rhodamine dye and ensure its efficient encapsulation and excellent optical properties inside polymer NPs. This work constitutes the first demonstration of an anion with $\pi$-delocalized negative charge capable to replace conventional weakly coordinating anions in preparation of dye-loaded polymer NPs. Importantly, the obtained anions are much less toxic than fluorinated tetraphenylborate, which enables preparation of much safer nanomaterials for bioimaging applications and increases their potential for clinical translation. Finally, a relatively simple chemistry of these anions opens the way to their further functionalization and scale up.
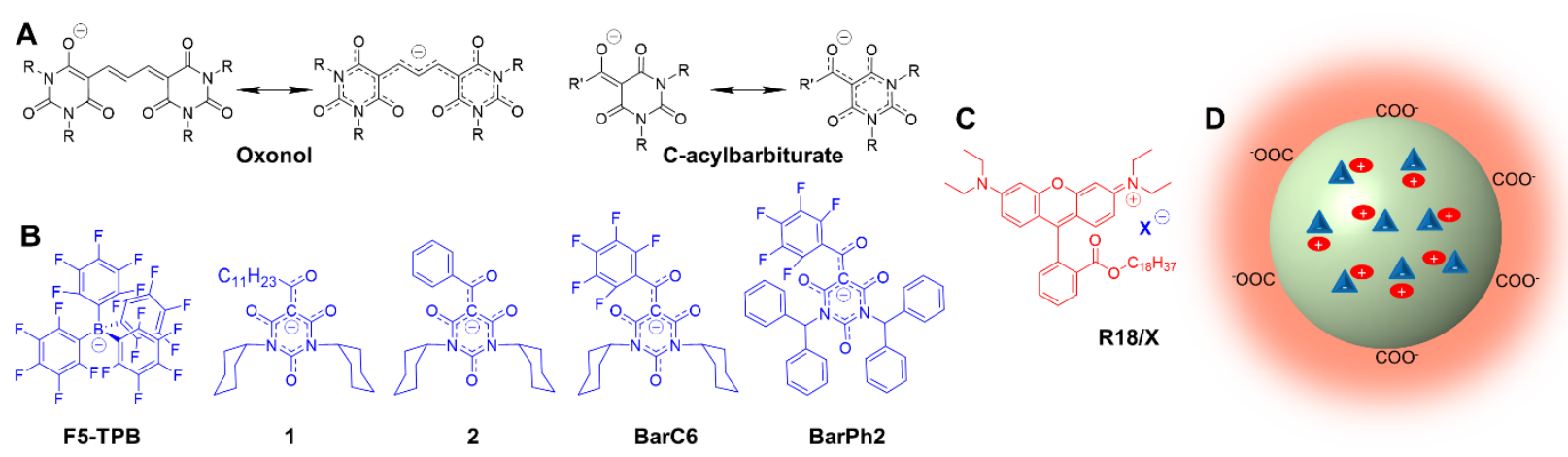

Figure 1. (A) Chemical structures of the oxonol dye scaffold and C-acyl barbiturates scaffolds with their resonance structures. (B-D) Schematic presentation of a fluorescent nanoparticle (D) encapsulated with ion pairs of a cationic dye R18 (C) and bulky hydrophobic counterions (B).

\section{Results and discussion}

Primarily, we synthesized barbiturates with (R) groups being cyclohexyl and R' being undecyl (1) or phenyl (2) (Figure 1B, Scheme 1). These molecules were readily obtained from the barbituric acid derivative and the corresponding carboxylic acids using EDC as coupling agent in the presence of DMAP. The obtained $C$-acyl barbiturates were mixed with octadecylrhodamine B (R18) perchlorate in DCM with excess of triethylamine for deprotonation of barbiturates and ion exchange. However, no stable enough ion pairs of compounds $\mathbf{1}$ and $\mathbf{2}$ with cationic dye R18 were formed to be purified by silica chromatography (Figure 1, Supporting Information). This result could be explained by insufficient electron-withdrawing effect of the used acyl groups, which made possible re-protonation of the formed enolate anion on silica, in line with relatively high calculated pKa of 6.51 and 6.04 for compounds $\mathbf{1}$ and $\mathbf{2}$, respectively. Therefore, to increase the 
acidity of the barbiturates, the R' group was changed to perfluorophenyl in molecule BarC6 (Figure 1B), which was obtained following the same methodology (Scheme 1). The anion of the obtained molecule could form ion pairs with the R18 cation that could be detected on silica thin layer chromatograph as a faster moving spot (Figure 1, Supporting Information), similarly to the ion pair R18/F5-TPB. We suppose that lower pKa values (estimated value 3.68) help to generate more stable ion pairs with the R18 dye. Further, we replaced the cyclohexyl (R) groups with diphenylmethyl to obtain the even bulkier perfluorophenyl analogue BarPh2 (Figure 1B, Scheme 1). The anionic form of $\mathrm{BarPh} 2$ also appeared to form stable ion pairs with R18 (Figure 1, Supporting Information), in line with similarly low calculated $\mathrm{pKa}$ values (3.98). Moreover, the $\mathrm{BarPh} 2$ salt migrated faster than that of BarC6, indicating a higher hydrophobicity of the former. Moreover, in contrast to the salt of BarC6, the BarPh2 salt did not show any trace of the R18 at the start of the TLC, indicating that it is more stable on silica.
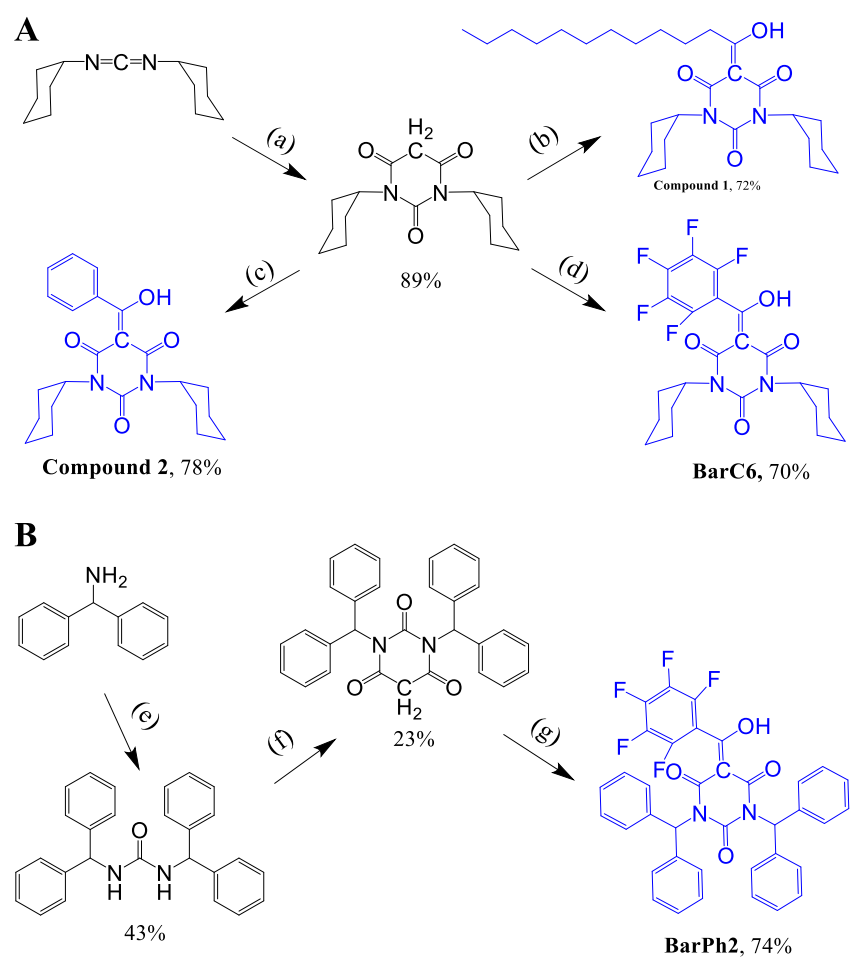

Scheme 1. Synthesis of target barbiturates (in blue) bearing N-cyclohexyl (A) and Ndiphenylmethyl (B) groups: (a) malonic acid, THF; (b) dodecanoic acid, EDC $\times \mathrm{HCl}$, DMAP, DCM; (c) benzoic acid, EDC $\times \mathrm{HCl}$, DMAP, DCM; (d) perfluorobenzoic acid, EDC $\times \mathrm{HCl}$, DMAP, DCM; (e) CDI, THF (f) malonyl chloride, DCM; (g) perfluorobenzoic acid, EDC $\times \mathrm{HCl}$, DMAP, DCM.

Both ion pairs of novel barbiturate anions with the R18 dye, namely R18/BarC6 and $\mathrm{R} 18 / \mathrm{BarPh} 2$, alongside with the already known $\mathrm{R} 18 / \mathrm{F} 5-\mathrm{TPB}$ and $\mathrm{R} 18 / \mathrm{ClO}_{4}$ as reference compounds, were encapsulated inside biodegradable PLGA (poly(lactide-co-glycolide)) 
nanoparticles (Figure 1) using a simple method based on nanoprecipitation from the watermiscible solvent acetonitrile. ${ }^{[15 a, 31]}$ The dyes were loaded at 5, 20 and $50 \mathrm{mM}$ concentration with respect to polymer.

The size of the obtained nanoparticles was measured by TEM (Figure 2) and DLS (Figure 3A). For both new barbiturates (BarC6 and BarPh2) at $50 \mathrm{mM}$ dye loading, TEM images revealed spherical shape of the obtained NPs, similarly to F5-TPB (Figure 2). The measured size values were similar for all three types of NPs in the range of 33-36 nm. By contrast, PLGA NPs loaded at $50 \mathrm{mM}$ with $\mathrm{R} 18$ and small hydrophilic perchlorate counterion $(\mathrm{R} 18 / \mathrm{ClO} 4)$ yielded large aggregates according to our earlier TEM data. ${ }^{[21]}$ DLS confirmed small size $(40-50 \mathrm{~nm})$ of the formulated PLGA NPs, which was independent of dye loading (Figure 3A). By contrast, ion pair $\mathrm{R} 18 / \mathrm{ClO} 4$ with the hydrophilic perchlorate counterion induced formation of much larger (>500 $\mathrm{nm}$ ) and colloidally unstable nanoparticles at $50 \mathrm{mM}$ dye loading. The latter observation, in line with our previous reports, ${ }^{[11 \mathrm{~b}, 15 \mathrm{a}]}$ can be explained by cationic dye adsorption at the anionic nanoparticle surface, which leads to decrease of surface charge and further NP aggregation. Zeta potential measurements showed strong negative values for PLGA NPs with all three studied bulky counterions at $50 \mathrm{mM}$ dye loading (Figure 2, Supporting Information). In contrast, the absolute value of the zeta potential was significantly lower in case of $\mathrm{R} 18 / \mathrm{ClO} 4$, confirming neutralization of the negative surface charge of NPs by cationic R18.

To evaluate quantitatively the dye encapsulation efficiency in NPs, we used our previously developed protocol - dialysis for $24 \mathrm{~h}$ of NPs solutions versus $1 \mathrm{mM}$ beta-cyclodextrin. ${ }^{[11 \mathrm{~b},}{ }^{21]}$ During this procedure all the non-encapsulated dyes and those adsorbed on the particle surface are washed off. Then, the comparison of dye concentration before and after dialysis gives us the encapsulation efficiency. The obtained results (Figure 3D) showed poor encapsulation efficiency of $\mathrm{R} 18 / \mathrm{ClO}_{4}(51 \pm 2 \%)$ and almost quantitative encapsulation of R18/F5-TPB (92 $\left.\pm 4 \%\right)$ at $50 \mathrm{mM}$ (corresponding to $\sim 7.5 \% \mathrm{w} / \mathrm{w}$ ) loading. For the barbiturate BarC6 anion, the dye encapsulation efficiency was $74 \pm 1 \%$, whereas for the bulkier and more hydrophobic $\mathrm{BarPh} 2$ the highest dye encapsulation efficiency was achieved $(96 \pm 1 \%)$. This efficient encapsulation of R18/BarPh2 can be explained by higher hydrophobicity of $\mathrm{BarPh} 2$ compared to its cyclohexyl-substituted rival BarC6. 

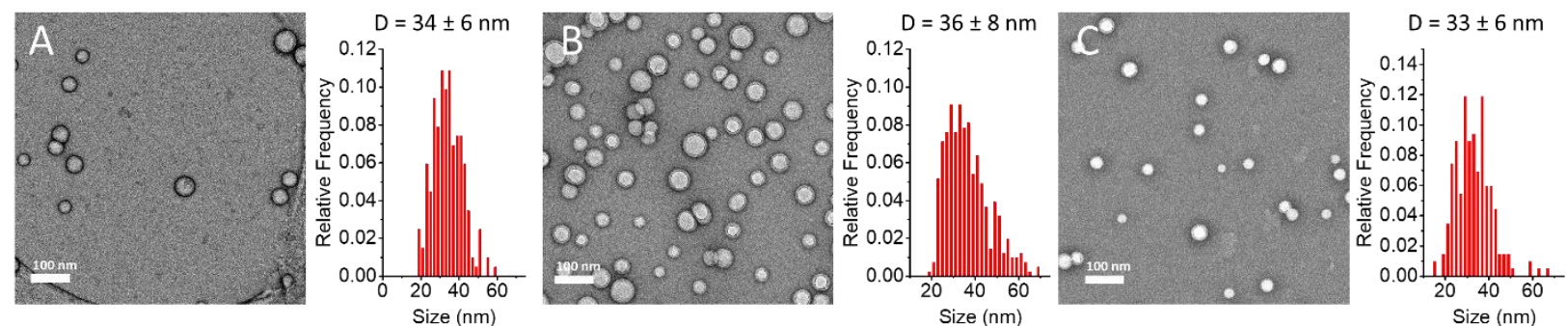

Figure 2. TEM images of NPs loaded with R18 dye with bulky counterions: F5-TPB (A), BarC6 (B) and $\mathrm{BarPh} 2(\mathrm{C})$. Graphs on the right of the TEM images correspond to size distribution histograms. The provided diameters are mean values of NPs based on analysis of $>200$ particles per condition. The error is the half-width at the half-maximum.

At $5 \mathrm{mM}$ dye loading, independently of the counterion, the quantum yields (QY) of all NPs were high (>70\%, Figure 3B). However, upon increase of loading to $50 \mathrm{mM}$, perchlorate demonstrated its inability to prevent dye self-quenching $(\mathrm{QY}=4 \%)$, in contrast to R18/F5-TPB loaded NPs, displaying a QY of 55\% at this loading. Remarkably, for the bulky barbiturate-based ion pair R18/BarPh2, the QY value was almost as high (52\%), suggesting that $\mathrm{BarPh} 2$ is as effective in preventing ACQ as the reference counterion F5-TPB. The ion pair R18/BarC6 displayed a QY of about $20 \%$ at $50 \mathrm{mM}$ loading, which was 5-fold higher compared to perchlorate, but still not as high as for F5-TPB. These results show that quantum yield and encapsulation efficiency of dye-loaded NPs correlate directly with bulkiness and hydrophobicity of the barbiturates, respectively, in line with our previous data. ${ }^{[32]}$
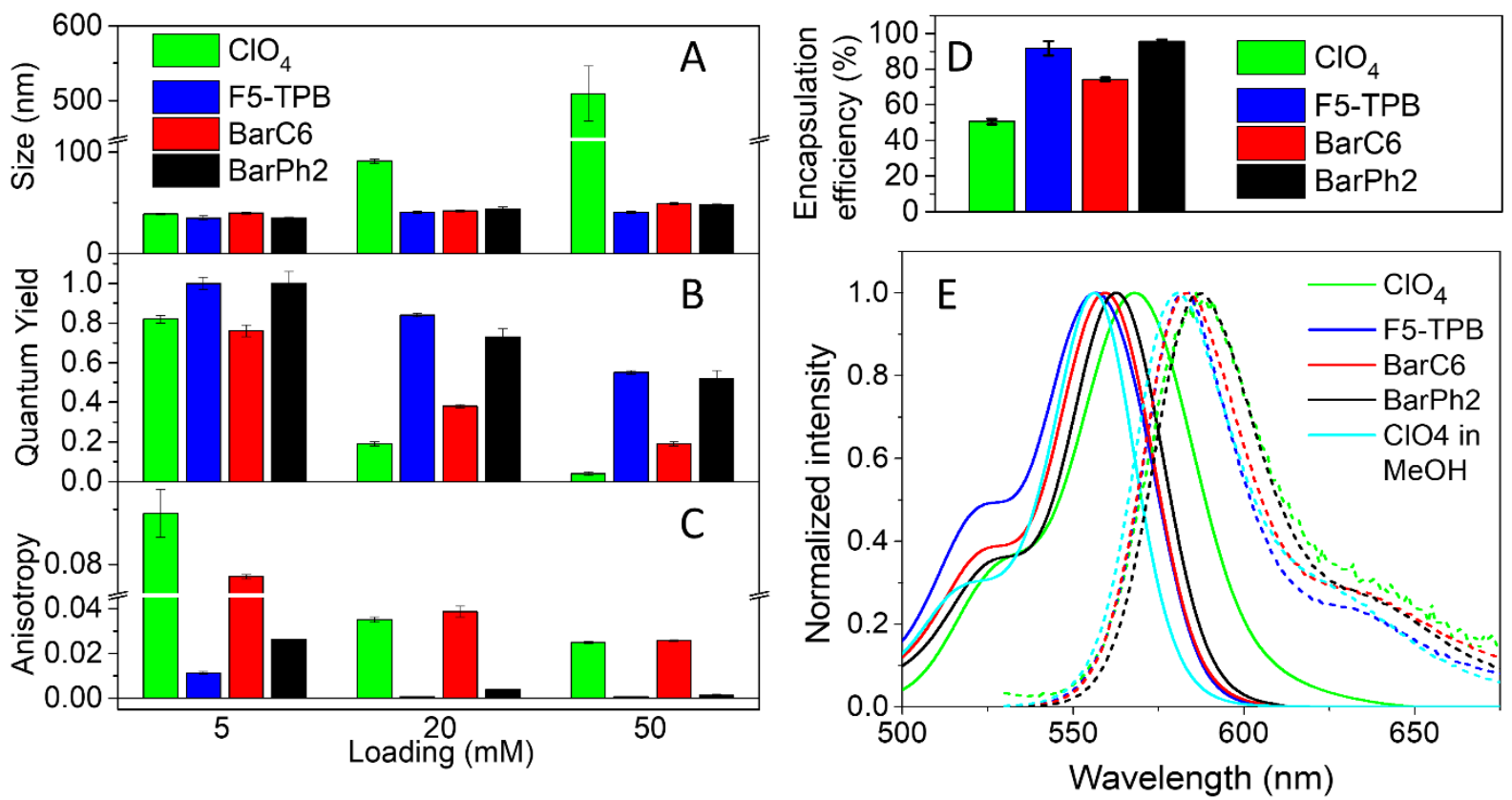

Figure 3. Properties of the developed NPs, loaded with ion pairs of R18 dye with different counterions. (A) Hydrodynamic diameter according to DLS, (B) fluorescence quantum yield, (C) fluorescence anisotropy and (D) encapsulation efficiency at $50 \mathrm{mM}$ dye loading. (E) Absorption 
and fluorescence spectra of NPs at $50 \mathrm{mM}$ dye loading and solution of $\mathrm{R} 18 / \mathrm{ClO}_{4}$ in methanol. Excitation wavelength was $520 \mathrm{~nm}$.

Fluorescence anisotropy measurement is one of the ways to study dye-dye communication inside NPs, asenergy transfer from dye to dye leads to loss of fluorescence anisotropy. ${ }^{[33]}$ According to our results, already at $5 \mathrm{mM}$ dye loading the fluorescence anisotropy values were very low (>0.01) for the F5-TPB counterion, in line with earlier data. ${ }^{[15 a]}$ In case of the BarPh2 counterion, the anisotropy was also relatively low at this loading (Figure 3C). It further decreased with dye loading reaching nearly zero at $50 \mathrm{mM}$ for both counterions, suggesting fast excitation energy transfer in these NPs. In sharp contrast, for the BarC6 counterion, the anisotropy values were relatively high at all loadings, similarly to those for the perchlorate counterion, implying that dye-dye communication inside NPs was weak. Thus, among the two barbiturates only the bulkier and more hydrophobic one $(\mathrm{BarPh} 2)$ favors dye-dye communication, similarly to F5-TPB.

The structure of the counterion also affected spectroscopic properties of the encapsulated R18 dye. Although at $5 \mathrm{mM}$ loading the effects in the absorption spectra were minor, at $50 \mathrm{mM}$ dye loading they became much more pronounced (Figure 3E). In line with previous studies, NPs loaded with R18/F5-TPB showed a blue-shifted absorption maximum compared to $\mathrm{R} 18 / \mathrm{ClO}_{4}-$ loaded NPs. The absorption maxima of R18 with barbiturate counterions were intermediate between $\mathrm{R} 18 / \mathrm{ClO}_{4}$ and $\mathrm{R} 18 / \mathrm{F} 5-\mathrm{TPB}$, though the BarPh2 was slightly red shifted compared to BarC6. Similar tendencies were observed in the fluorescence spectra. The red shifted absorption and emission of the BarPh2 is unexpected, given its similar performance with F5-TPB. One could speculate that, unlike the case of F5-TPB, where negative charge is mostly localized at weakly polarizable fluorine atoms, $\mathrm{BarPh} 2$, due to its higher electronic polarizability decreases the energy of the excited state of R18. 

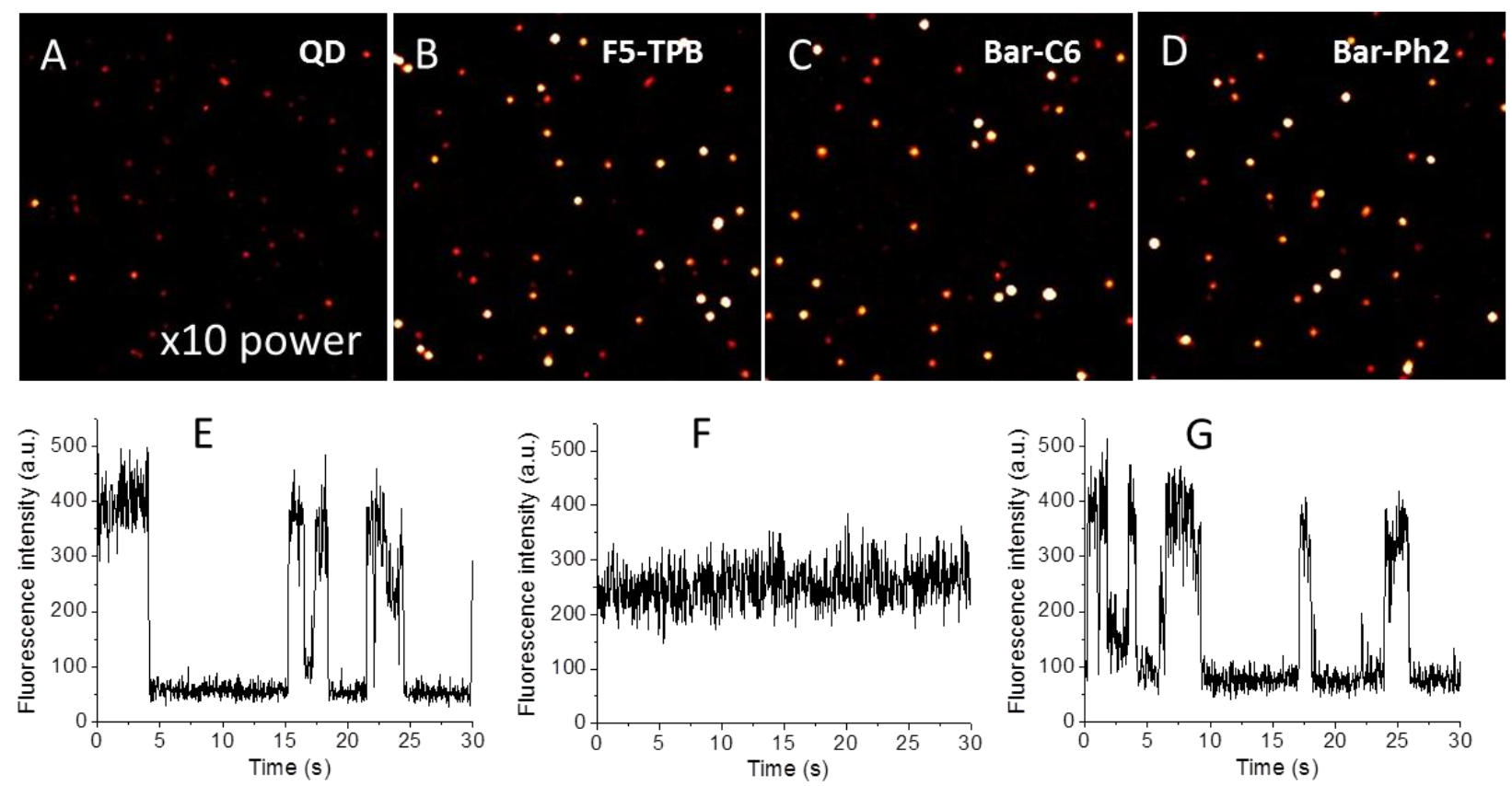

Figure 4. Single-particle fluorescence properties of the tested dye-loaded PLGA NPs. (A-D) Wide-field fluorescence images of QD585 (A), R18/F5-TPB (B), R18/BarC6 (C) and R18/BarPh2 (D) NPs at $50 \mathrm{mM}$ dye loading. Identical imaging conditions were applied for dye-loaded NPs (excitation power density of $4.4 \mathrm{~W} / \mathrm{cm}^{2}$ at $550 \mathrm{~nm}$ ), ten times higher illumination power was used for QDs. Image size was $15 \times 15 \mu \mathrm{m}$. (E-G) Typical single-particle fluorescence traces of NPs loaded with R18/F5-TPB (E), R18/BarC6 (F) and R18/BarPh2 (G), recorded at the same conditions.

Single-particle brightness was studied by wide-field microscopy. NPs loaded at $50 \mathrm{mM}$ with $\mathrm{R} 18 / \mathrm{BarPh} 2, \mathrm{R} 18 / \mathrm{BarC6}$ and R18/F5-TPB as well as commercial quantum dots of similar emission range, QD585, were immobilized on glass and excited by a LED source at $550 \mathrm{~nm}$ (Figure 4A-D). All of the tested dye-loaded NPs appeared much brighter than quantum dots QD585: R18/F5-TPB, R18/BarC6 and R18/BarPh2-loaded NPs were, respectively, 33, 29 and 28fold brighter. Surprisingly, the brightness of R18/BarC6 NPs (Figure 4C) was close to those of R18/F5-TPB and R18/BarPh2 NPs (Figure 4B, D), despite having more than two-fold lower quantum yield and lower encapsulation efficiency. This peculiar behavior of these NPs can be explained based on single-particle fluorescence intensity traces (Figure 4E-G). R18/BarPh2 NPs (Figure 4G) underwent ON/OFF fluorescence switching (blinking), similarly to R18/F5-TPB NPs (Figure 4E). Statistical analysis based on the mean of three measurements ( $>400$ particles of each type) suggested that blinking took place for $95+/-0.5 \%$ and $78+/-2 \%$ of all recorded R18/F5-TPB and R18/BarPh2 NPs, respectively. According to earlier studies of R18/F5-TPB NPs, such behavior is only possible when the dyes are in close proximity inside the nanoparticle, making ultrafast energy transfer possible and in consequence effective quenching of the whole particle by transient dark species (such as triplet/radical states of rhodamine). ${ }^{[9,15 \mathrm{a}]}$ Thus, similarly to F5-TPB, the $\mathrm{BarPh} 2$ anion may favor clustering of $\mathrm{R} 18$ dyes inside the polymer matrix, which is in 
agreement with the observed low fluorescence anisotropy values (Figure 3C). By contrast, the majority (76 $\pm 6 \%$ ) of R18/BarC6 NPs particles showed constant emission without blinking (Figure 4F). This very different behavior is probably related to inefficiency of dye-dye energy transfer, as suggested by relatively high values of the fluorescence anisotropy. Thus, higher than expected relative brightness of R18/BarC6 NPs could be explained by poor dye-dye communication, which prevents quenching the encapsulated dyes by a single dark states or by singlet-singlet annihilation. We can speculate that poor dye-dye communication for R18/BarC6 even at relatively high loading originates from rather homogeneous distribution of fluorophores within the polymer matrix of NPs, whereas bulkier and more hydrophobic R18/BarPh2 tends to cluster. In our previous work, we observed similar effects in the fluorescence anisotropy and particle blinking when bulky fluorinated TPBs were compared to the much smaller anion $\mathrm{B}\left(\mathrm{CF}_{3}\right)_{4} \cdot{ }^{[11 \mathrm{~b}]}$ Together with the present data, we have now a solid background to conclude that size and hydrophobicity of the counterion is a tool to control dye clustering in the polymer matrix of NPs. We expect that larger and more hydrophobic counterions favor dye clustering, because their salts with R18 are significantly more hydrophobic than the PLGA polymer, so that during nanoprecipitation they precipitate before the polymer, forming the core with clustered dye. In contrast, R18 salts with counterions of intermediate hydrophobicity (like BarC6 and $\left.\mathrm{B}\left(\mathrm{CF}_{3}\right)_{4}\right)$ would co-precipitate together with the polymer, thus favoring more homogeneous dye distribution in the polymer with longer dye-dye distances, leading to higher fluorescence anisotropy and stable emission without blinking.

To test the obtained NPs in biological media, they were incubated with KB cells for 3 hours and then the cells were washed and imaged by confocal microscopy. All four counterions at the highest $(50 \mathrm{mM})$ and lowest $(5 \mathrm{mM})$ dye loading were tested (Figure 5). R18/F5-TPB NPs at 5 $\mathrm{mM}$ loading (Figure 5B) appeared as bright dots, being localized in the perinuclear regions, and without clear background signal from other parts of the cells. This result is in line with previous data, confirming that after endocytosis of R18/F5-TPB NPs, no significant dye leakage is observed. R18/BarPh2 and R18/BarC6 NPs at this loading showed similar behavior (Figure 5D and $4 \mathrm{C}$, respectively). A significant degree of leakage was observed for $\mathrm{R} 18 / \mathrm{ClO}_{4} \mathrm{NPs}$ at $5 \mathrm{mM}$ loading (Figure 5A) as a diffuse fluorescence inside the cells, so that the characteristic perinuclear dots (endosomes/lysosomes) were practically not visible. The same tendency was observed at 50 $\mathrm{mM}$ loading, where $\mathrm{R} 18 / \mathrm{ClO}_{4} \mathrm{NPs}$ showed strong fluorescence all over the cells, while for NPs with other ion pairs, a clear dotted fluorescence was still observed (Figure 5E-H). Nevertheless, for both R18/F5-TPB and R18/BarC6 NPs at $50 \mathrm{mM}$ loading some diffuse intracellular fluorescence started to be visible (Figures $5 \mathrm{~F}$ and $5 \mathrm{G}$, respectively), indicating imperfect encapsulation of these ion pairs at the highest loading. Remarkably, for $50 \mathrm{mM} \mathrm{R} 18 / \mathrm{BarPh} 2 \mathrm{NPs}$, diffuse fluorescence was negligible (Figure 5H), suggesting absence of dye leakage in biological 
media. The observed cellular data correlated well with the encapsulation studies, showing the highest encapsulation efficiency for $\mathrm{R} 18 / \mathrm{BarPh} 2$ and the lowest for $\mathrm{R} 18 / \mathrm{ClO}_{4}$ (Figure 3D). Thus, the novel counterion BarPh2 outperforms our "golden standard" F5-TPB for bioimaging purposes. In this respect, it is close to the recently reported $\mathrm{F} 9-\mathrm{Al}$, a strongly fluorinated aluminium-based counterion, ${ }^{[21]}$ showing superior encapsulation of the R18 dye.

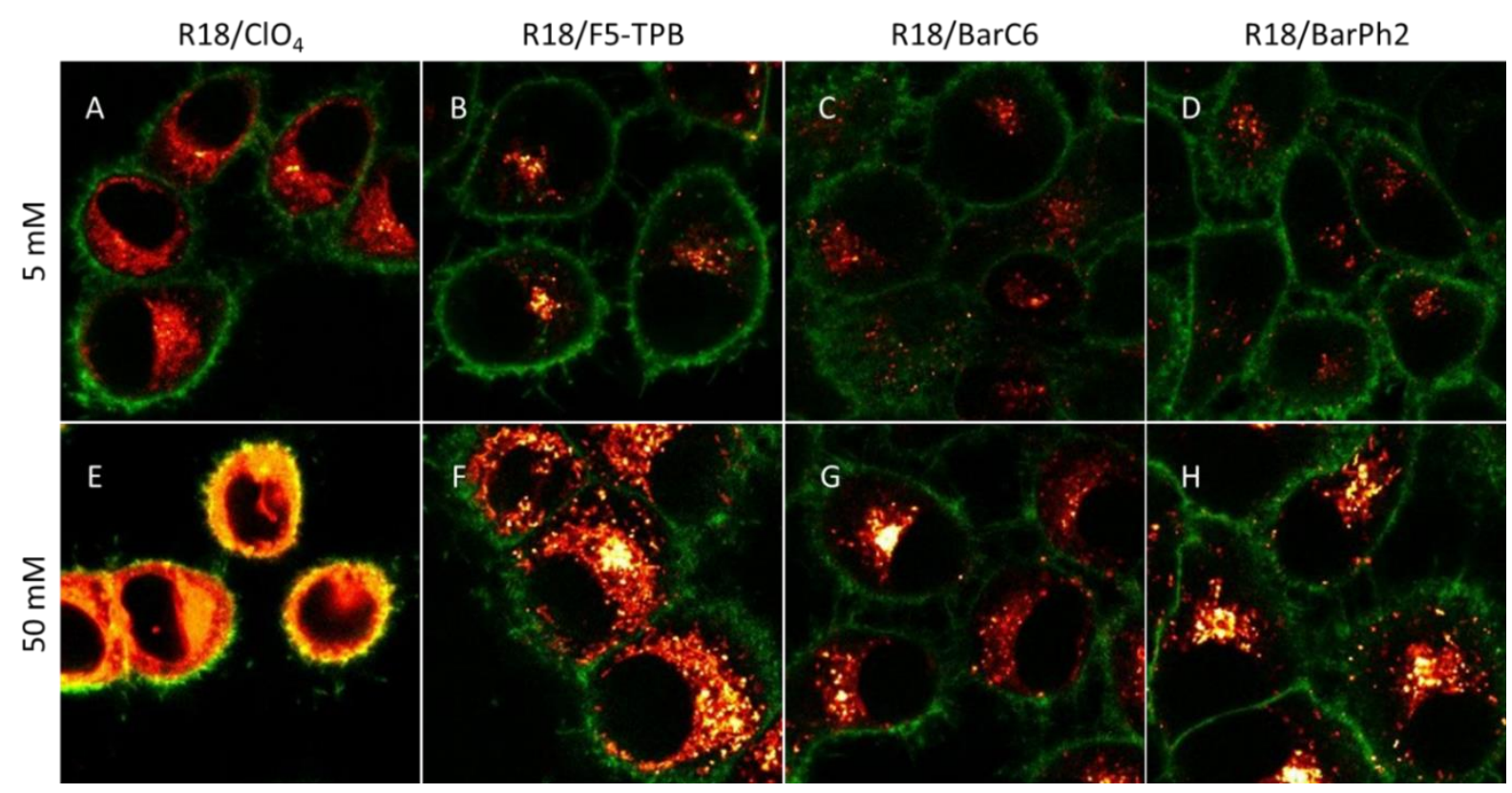

Figure 5. Confocal images of KB cells incubated for $3 \mathrm{~h}$ with dye-loaded PLGA NPs. NPs were loaded with R18/ClO4, R18/F5-TPB, R18/BarC6 and R18/BarPh2 at 5 and $50 \mathrm{mM}$ concentrations. Excitation wavelength was $561 \mathrm{~nm}$, emission was collected from 570 to $650 \mathrm{~nm}$. Image size was $58.4 \times 58.4 \mu \mathrm{m}$.

Finally, we compared the cytotoxicity of the counterions vs our standard F5-TPB. To this end, we prepared salts of BarC6 and BarPh2 with 1.2 eq. of triethylamine and studied them together with the F5-TPB lithium salt in HeLa cells using standard MTT assay. After $24 \mathrm{~h}$ incubation, the F5-TPB counterion was found to be the most cytotoxic, showing $<50 \%$ of cell viability at $3 \mu \mathrm{M}$ concentration (Figure 6). In sharp contrast, both barbiturates demonstrated virtually no cytotoxicity at this concentration. Moreover, BarPh2 showed low cytotoxicity with cell viability $>50 \%$ for the studied concentration range up to $30 \mu \mathrm{M}$. BarC6, having lower hydrophobicity and slightly lower pKa than $\mathrm{BarPh} 2$, displayed intermediate cytotoxicity between F5-TPB and BarPh2, with cell viability $<50 \%$ at $10 \mu \mathrm{M}$ concentration. Thus, both barbiturates, especially BarPh2, are less cytotoxic than our standard F5-TPB, which is probably connected to the lower capacity of barbiturates to transport protons and other cations through biological membranes. We can speculate that barbiturates due to their high hydrophobicity and relatively weak acidity (3.68 and 3.98) are bound to lipid membranes in molecular form which prevents them 
from deprotonation. The fact that the more hydrophobic $\mathrm{BarPh} 2$ is much less toxic than BarC6 supports this hypothesis, because the former should have even stronger partitioning into lipids, further decreasing the probability of its deprotonation. By contrast, F5-TPB can preserve the anionic form when bound to the lipid membrane and therefore can operate as an efficient shuttle of protons and other cations.

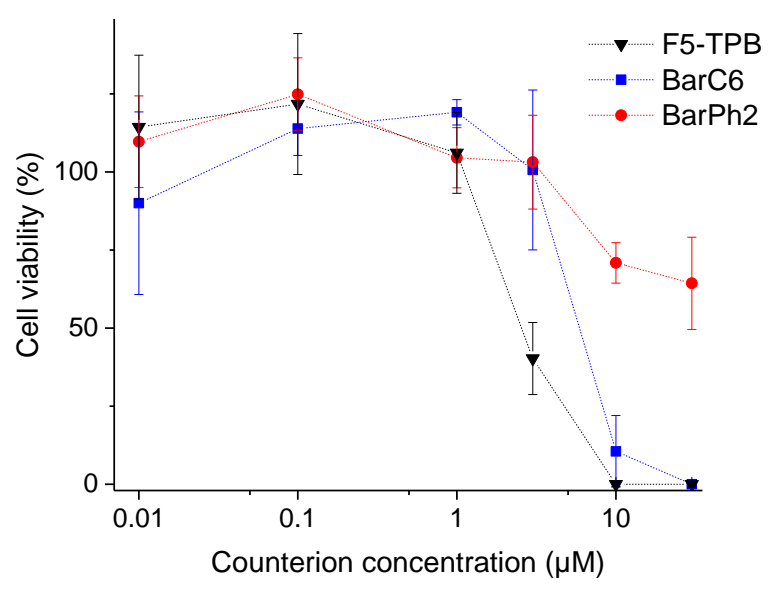

Figure 6. Viability of HeLa cells exposed for $24 \mathrm{~h}$ to different concentrations of the three counterion salts: F5-TPB lithium salt, BarC6 and BarPh2 salts with triethylamine (1.2 eq.). Cell viability was tested in quadruplicate and repeated at least two times using MTT. The error bars correspond to the standard deviation.

\section{Conclusions}

In conclusion, we describe a new family of hydrophobic anions based on acylated barbiturates with strongly delocalized negative charge, which, similarly to weakly coordinating anions based on boron or aluminium, can enhance encapsulation and emission of cationic dyes inside polymer NPs. Among the studied acyl barbiturates, the bulkiest and the most hydrophobic anion prevents self-quenching of a cationic rhodamine B derivative as efficiently as the "golden standard" tetrakis(pentafluorophenyl)borate and at the same time enables more efficient dye encapsulation with minimal leakage in living cells. Moreover, the chemical structure (hydrophobicity) of the barbiturate counterion can control the dye-dye communication, as evidenced by fluorescence anisotropy and, thus, tune the emission mode of nanoparticles from complete on/off switching (blinking) to steady emission. Finally, the acyl barbiturates displayed significantly lower cytotoxicity compared to tetrakis(pentafluorophenyl)borate. Moreover, the least toxic acyl barbiturate $\mathrm{BarPh} 2$ was both the more hydrophobic and slightly less acidic one, which is probably related to its inability to get deprotonated and thus to transfer the proton and other cations through biological membranes. These results provide insights on molecular design of bulky hydrophobic counterions with minimized cytotoxicity. Overall, barbiturate anions, owing 
to these unique properties, open the route to preparation of non-toxic and eco-friendly dye-loaded polymer NPs, which can find application in biosensing, bioimaging and materials science.

\section{Experimental Section}

Detailed protocols of the synthesis and characterization of barbiturates, preparation and characterization of NPs, their studies in cells as well as some data on TLC of the ion pairs and zeta potential of NPs are provided in the Supporting Information.

\section{Acknowledgements}

This work was supported by the European Research Council ERC Consolidator grant BrightSens 648528. B.A. was supported by LabEx Chimie des Systèmes Complexes. I.O.A. acknowledges support from Marie Curie post-doctoral research grant (H2020-MSCA-IF-2018, DNANanoProbes 846571 ) by the European Union.

\section{Conflict of Interest}

The authors declare no conflict of interest.

\section{References}

[1] a) O. S. Wolfbeis, Chem. Soc. Rev. 2015, 44, 4743; b) N. Hildebrandt, C. M. Spillmann, W. R. Algar, T. Pons, M. H. Stewart, E. Oh, K. Susumu, S. A. Díaz, J. B. Delehanty, I. L. Medintz, Chemical Reviews 2017, 117, 536; c) P. D. Howes, R. Chandrawati, M. M. Stevens, Science 2014, 346, 53.

[2] Y. F. Jiang, J. McNeill, Chemical Reviews 2017, 117, 838.

[3] a) J. Mei, N. L. C. Leung, R. T. K. Kwok, J. W. Y. Lam, B. Z. Tang, Chemical Reviews 2015, 115, 11718; b) K. Li, B. Liu, Chem. Soc. Rev. 2014, 43, 6570.

[4] a) L. Feng, C. Zhu, H. Yuan, L. Liu, F. Lv, S. Wang, Chem. Soc. Rev. 2013, 42, 6620 ; b) C. Wu, D. T. Chiu, Angewandte Chemie International Edition 2013, 52, 3086.

[5] a) A. Reisch, A. S. Klymchenko, Small 2016, 12, 1968; b) S. Y. Ong, C. Zhang, D. Xiao, S. Q. Yao, Angewandte Chemie International Edition 2021, DOI: 10.1002/anie.202101964.

[6] A. S. Klymchenko, F. Liu, M. Collot, N. Anton, Advanced Healthcare Materials 2021, 10, 2001289.

[7] a) F. Danhier, E. Ansorena, J. M. Silva, R. Coco, A. Le Breton, V. Preat, J. Control. Release 2012, 161, 505; b) A. Kumari, S. K. Yadav, S. C. Yadav, Colloid Surf. BBiointerfaces 2010, 75, 1.

[8] B. Andreiuk, A. Reisch, M. Lindecker, G. Follain, N. Peyriéras, J. G. Goetz, A. S. Klymchenko, Small 2017, 13, 1701582.

[9] A. Reisch, K. Trofymchuk, A. Runser, G. Fleith, M. Rawiso, A. S. Klymchenko, ACS Applied Materials \& Interfaces 2017, 9, 43030.

[10] K. Trofymchuk, A. Reisch, I. Shulov, Y. Mély, A. S. Klymchenko, Nanoscale 2014, 6, 12934. 
[11] a) S. Snipstad, et al., Cytometry $A$ 2017, 91, 760; b) K. Trofymchuk, J. Valanciunaite, B. Andreiuk, A. Reisch, M. Collot, A. S. Klymchenko, J Mater Chem B 2019, 7, 5199.

[12] F. Wurthner, T. E. Kaiser, C. R. Saha-Moller, Angew. Chem.-Int. Edit. 2011, 50, 3376.

[13] W. Qin, D. Ding, J. Z. Liu, W. Z. Yuan, Y. Hu, B. Liu, B. Z. Tang, Advanced Functional Materials 2012, 22, 771.

[14] B. Zhang, H. Soleimaninejad, D. J. Jones, J. M. White, K. P. Ghiggino, T. A. Smith, W. W. H. Wong, Chemistry of Materials 2017, 29, 8395.

[15] a) A. Reisch, P. Didier, L. Richert, S. Oncul, Y. Arntz, Y. Mély, A. S. Klymchenko, Nature Communications 2014, 5, 4089; b) C. R. Benson, et al., Chem 2020, 6, 1978.

[16] I. Krossing, I. Raabe, Angew. Chem. Int. Ed. 2004, 43, 2066.

[17] T. A. Engesser, M. R. Lichtenthaler, M. Schleep, I. Krossing, Chem. Soc. Rev. 2016, 45, 789.

[18] A. B. A. Rupp, I. Krossing, Accounts Chem. Res. 2015, 48, 2537.

[19] a) D. K. Bwambok, B. El-Zahab, S. K. Challa, M. Li, L. Chandler, G. A. Baker, I. M. Warner, Acs Nano 2009, 3, 3854; b) P. Magut, S. Das, V. Fernand, J. Losso, K. McDonough, B. Naylor, S. Aggarwal, I. Warner, Journal of the American Chemical Society 2013, 135, 15873; c) H. Yao, K. Ashiba, Rsc Advances 2011, 1, 834; d) T. Funada, T. Hirose, N. Tamai, H. Yao, Phys. Chem. Chem. Phys. 2015, 17, 11006.

[20] I. Shulov, S. Oncul, A. Reisch, Y. Arntz, M. Collot, Y. Mely, A. S. Klymchenko, Nanoscale 2015, 7, 18198.

[21] B. Andreiuk, A. Reisch, V. G. Pivovarenko, A. S. Klymchenko, Materials Chemistry Frontiers 2017, 1, 2309.

[22] I. O. Aparin, N. Melnychuk, A. S. Klymchenko, Advanced Optical Materials 2020, 8, 2000027.

[23] N. Melnychuk, S. Egloff, A. Runser, A. Reisch, A. S. Klymchenko, Angewandte Chemie International Edition 2020, 59, 6811.

[24] N. Melnychuk, A. S. Klymchenko, Journal of the American Chemical Society 2018, 140, 10856.

[25] K. Trofymchuk, A. Reisch, P. Didier, F. Fras, P. Gilliot, Y. Mely, A. S. Klymchenko, Nature Photonics 2017, 11, 657.

[26] I. Khalin, D. Heimburger, N. Melnychuk, M. Collot, B. Groschup, F. Hellal, A. Reisch, N. Plesnila, A. S. Klymchenko, ACS Nano 2020, 14, 9755.

[27] W. Michael Arnold, U. Zimmermann, W. Heiden, J. Ahlers, Biochimica et Biophysica Acta (BBA) - Biomembranes 1988, 942, 96.

[28] a) S. Fischer, J. Schmidt, P. Strauch, A. Thomas, Angewandte Chemie International Edition 2013, 52, 12174; b) A. Franzke, A. Pfaltz, Chemistry - A European Journal 2011, 17, 4131.

[29] J. Chen, S. M. A. Fateminia, L. Kacenauskaite, N. Bærentsen, S. G. Stenspil, J. Bredehoeft, K. L. Martinez, A. H. Flood, B. W. Laursen, Angewandte Chemie International Edition 2021, 60, 9450.

[30] J. E. Gonzalez, R. Y. Tsien, Chem. Biol. 1997, 4, 269.

[31] A. Reisch, A. Runser, Y. Arntz, Y. Mély, A. S. Klymchenko, ACS Nano 2015, 9, 5104.

[32] B. Andreiuk, A. Reisch, E. Bernhardt, A. S. Klymchenko, Chem. Asian J. 2019, 14, 836.

[33] a) K. A. Colby, J. J. Burdett, R. F. Frisbee, L. Zhu, R. J. Dillon, C. J. Bardeen, Journal of Physical Chemistry A 2010, 114, 3471; b) C. J. Bardeen, in Annual Review of Physical Chemistry, Vol 65, Vol. 65 (Eds.: M. A. Johnson, T. J. 
Martinez), 2014, pp. 127; c) R. Camacho, D. Tauber, I. C. Scheblykin, Advanced Materials 2019, 31, 1805671. 


\section{Entry for the Table of Contents}

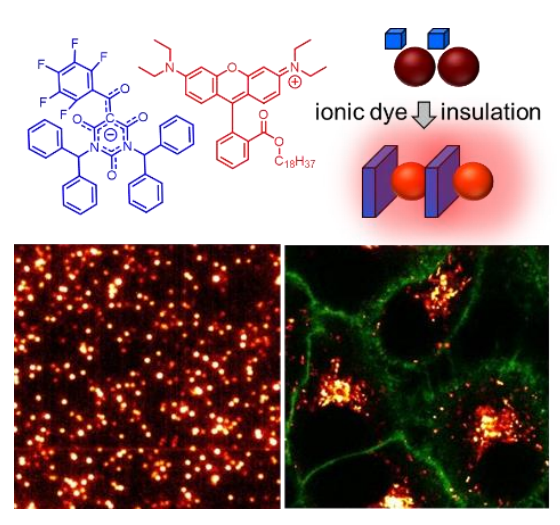

Bulky hydrophobic anions based on fluorinated $C$-acyl barbiturates with delocalized negative charge are found to provide insulation of ionic dyes, thus preventing their aggregation-caused quenching in polymeric nanoparticles. Their chemical structure defines dye encapsulation, particle brightness and on/off switching. Compared to currently used anions, $C$-acyl barbiturates are nontoxic, while offering chemical availability and versatility. 